\title{
Approximating common fixed points of averaged self-mappings with applications to the split feasibility problem and maximal monotone operators in Hilbert spaces
}

Young-Ye Huang ${ }^{1}$ and Chung-Chien Hong ${ }^{2 *}$

*Correspondence:

chong@mail.npust.edu.tw

${ }^{2}$ Department of Industrial

Management, National Pingtung

University of Science and

Technology, 1 Shuefu Rd., Neipu,

Pingtung, 91201, Taiwan

Full list of author information is

available at the end of the article

\begin{abstract}
In this paper, a modified proximal point algorithm for finding common fixed points of averaged self-mappings in Hilbert spaces is introduced and a strong convergence theorem associated with it is proved. As a consequence, we apply it to study the split feasibility problem, the zero point problem of maximal monotone operators, the minimization problem and the equilibrium problem, and to show that the unique minimum norm solution can be obtained through our algorithm for each of the aforementioned problems. Our results generalize and unify many results that occur in the literature.
\end{abstract}

MSC: 47H10; 47J25; 68W25

Keywords: averaged mapping; firmly nonexpansive mapping; maximal monotone operator; split feasibility problem; minimization problem; equilibrium problem

\section{Introduction}

Throughout this paper, $\mathcal{H}$ denotes a real Hilbert space with the inner product $\langle\cdot, \cdot\rangle$ and the norm $\|\cdot\|, I$ the identity mapping on $\mathcal{H}, \mathbb{N}$ the set of all natural numbers and $\mathbb{R}$ the set of all real numbers. For a self-mapping $T$ on $\mathcal{H}, F(T)$ denotes the set of all fixed points of $T$.

Let $C$ and $Q$ be nonempty closed convex subsets of two Hilbert spaces $\mathcal{H}_{1}$ and $\mathcal{H}_{2}$ respectively, and let $A: \mathcal{H}_{1} \rightarrow \mathcal{H}_{2}$ be a bounded linear mapping. The split feasibility problem $(\mathrm{SFP})$ is the problem of finding a point with the property:

$$
x^{*} \in C \text { and } A x^{*} \in Q
$$

The SFP was first introduced by Censor and Elfving [1] for modeling inverse problems which arise from phase retrievals and medical image reconstruction. Recently, it has been found that the SFP can also be used to model the intensity-modulated radiation therapy. For details, the readers are referred to $\mathrm{Xu}$ [2] and the references therein.

Assume that the SFP has a solution. There are many iterative methods designed to approximate its solutions. The most popular algorithm is the $C Q$ algorithm introduced by Byrne [3, 4]:

C) 2013 Huang and Hong: licensee Springer. This is an Open Access article distributed under the terms of the Creative Commons Attribution License (http://creativecommons.org/licenses/by/2.0), which permits unrestricted use, distribution, and reproduction in any medium, provided the original work is properly cited. 
It starts with any $x_{1} \in \mathcal{H}_{1}$ and generates a sequence $\left\{x_{n}\right\}$ through the iteration

$$
x_{n+1}=P_{C}\left(I-\gamma A^{*}\left(I-P_{Q}\right) A\right) x_{n},
$$

where $\gamma \in\left(0, \frac{2}{\|A\|^{2}}\right), A^{*}$ is the adjoint of $A, P_{C}$ and $P_{Q}$ are the metric projections onto $C$ and $Q$ respectively.

The sequence $\left\{x_{n}\right\}$ generated by the $C Q$ algorithm (2) converges weakly to a solution of SFP (1), cf. [2-4]. Under the assumption that SFP (1) has a solution, it is known that a point $x^{*} \in \mathcal{H}_{1}$ solves the SFP (1) if and only if $x^{*}$ is a fixed point of the operator

$$
P_{C}\left(I-\gamma A^{*}\left(I-P_{Q}\right) A\right)
$$

$c f .[2]$, where $\mathrm{Xu}$ also proposed the regularized method

$$
x_{n+1}=P_{C}\left(I-\gamma_{n}\left(A^{*}\left(I-P_{Q}\right) A+\alpha_{n} I\right)\right) x_{n},
$$

and proved that the sequence $\left\{x_{n}\right\}$ converges strongly to a minimum norm solution of SFP (1) provided the parameters $\left\{\alpha_{n}\right\}$ and $\left\{\gamma_{n}\right\}$ verify some suitable conditions. This regularized method was further investigated by Yao, Jiang and Liou [5], and Yao, Liou and Shahzad [6].

Motivated by the above works, it is desirable to devise an algorithm for approximating a point $x^{*} \in C$ so that

$$
A x^{*} \in Q \text { and } B x^{*} \in Q \text {, }
$$

where $A, B$ are two bounded linear mappings from $\mathcal{H}_{1}$ to $\mathcal{H}_{2}$.

On the other hand, it has been an interesting topic of finding zero points of maximal monotone operators. A set-valued map $A: \mathcal{H} \rightarrow 2^{\mathcal{H}}$ with the domain $\mathcal{D}(A)$ is called monotone if

$$
\langle x-y, u-v\rangle \geq 0
$$

for all $x, y \in \mathcal{D}(A)$ and for any $u \in A(x), v \in A(y)$, where $\mathcal{D}(A)$ is defined to be

$$
\mathcal{D}(A)=\{x \in \mathcal{H}: A x \neq \varnothing\}
$$

$A$ is said to be maximal monotone if its graph $\{(x, u): x \in \mathcal{H}, u \in A(x)\}$ is not properly contained in the graph of any other monotone operator. For a positive real number $\alpha$, we denote by $J_{\alpha}^{A}$ the resolvent of a monotone operator $A$, that is, $J_{\alpha}^{A}(x)=(I+\alpha A)^{-1}(x)$ for any $x \in \mathcal{H}$. A point $v \in \mathcal{H}$ is called a zero point of a maximal monotone operator $A$ if $0 \in A(v)$. In the sequel, we shall denote the set of all zero points of $A$ by $A^{-1} 0$, which is equal to $F\left(J_{\alpha}^{A}\right)$ for any $\alpha>0$. A well-known method to solve this problem is the proximal point algorithm which starts with any initial point $x_{1} \in \mathcal{H}$ and then generates the sequence $\left\{x_{n}\right\}$ in $\mathcal{H}$ by

$$
x_{n+1}=J_{\alpha_{n}}^{A} x_{n}, \quad n \in \mathbb{N},
$$

where $\left\{\alpha_{n}\right\}$ is a sequence of positive real numbers. This algorithm was first introduced by Martinet [7] and then generally studied by Rockafellar [8], who devised the iterative 
sequence $\left\{x_{n}\right\}$ by

$$
x_{n+1}=J_{\alpha_{n}}^{A} x_{n}+e_{n}, \quad n \in \mathbb{N}
$$

where $\left\{e_{n}\right\}$ is an error sequence in $\mathcal{H}$. Rockafellar showed that the sequence $\left\{x_{n}\right\}$ generated by (6) converges weakly to an element of $A^{-1} 0$ provided that $A^{-1} 0 \neq \varnothing$ and $\liminf _{n \rightarrow \infty} \alpha_{n}>0$. In 1991, Güler [9] established an example showing that the sequence $\left\{x_{n}\right\}$ generated by (6) converges weakly but not strongly. Since then, many authors have conducted research on modifying the sequence in (6) so that the strong convergence is guaranteed, $c f$. [10-19] and the references therein. Recently, Wang and Cui [16] considered the following algorithm:

$$
x_{n+1}=a_{n} u+b_{n} x_{n}+c_{n} J_{\alpha_{n}}^{A} x_{n}+e_{n}, \quad n \in \mathbb{N},
$$

where $\left\{a_{n}\right\},\left\{b_{n}\right\},\left\{c_{n}\right\}$ are sequences in $(0,1)$ with $a_{n}+b_{n}+c_{n}=1$ for all $n \in \mathbb{N}$, and $\left\{e_{n}\right\}$ is an error sequence in $\mathcal{H}$. They showed that the sequence $\left\{x_{n}\right\}$ generated by (7) converges strongly to a zero point of $A$ provided the following conditions (i) and (ii) are verified:

$$
\begin{aligned}
& \text { (i) } \lim _{n \rightarrow \infty} a_{n}=0, \quad \sum_{n=1}^{\infty} a_{n}=\infty, \quad \liminf _{n \rightarrow \infty} c_{n}>0, \quad \liminf _{n \rightarrow \infty} \alpha_{n}>0 \text {; } \\
& \text { (ii) either } \sum_{n=1}^{\infty}\left\|e_{n}\right\|<\infty \text { or } \quad \lim _{n \rightarrow \infty} \frac{\left\|e_{n}\right\|}{a_{n}}=0 .
\end{aligned}
$$

This theorem generalizes and unifies many results that occur in the literature, $c f$. [10-12, $18,20]$.

For another maximal monotone operator $B$, we would like to seek appropriate conditions on the coefficient sequences $\left\{a_{n}\right\},\left\{b_{n}\right\},\left\{c_{n}\right\}$ and $\left\{d_{n}\right\}$ so that the sequence $\left\{x_{n}\right\}$ generated by

$$
x_{n+1}=a_{n} u+b_{n} J_{\beta_{n}}^{B} x_{n}+c_{n} J_{\alpha_{n}}^{A} x_{n}+d_{n} e_{n}, \quad n \in \mathbb{N},
$$

can converge strongly to a common zero of $A$ and $B$.

We find that both of problems (5) and (8) can be solved simultaneously in a more general setting. As a matter of fact, any resolvent is firmly nonexpansive and any firmly nonexpansive mapping is $\frac{1}{2}$-averaged, $c f$. [21], which is a special case of $\lambda$-averaged mappings (for the definition of $\lambda$-averaged mappings, we refer readers to Section 2). Also, as shown in the proof of Theorem 3.6 of [2], for any $\gamma \in \mathbb{R}$ with $0<\gamma<\frac{2}{\|A\|^{2}}$, the operator (3) is $\frac{2+\gamma\|A\|^{2}}{4}$ averaged. It is quite natural to ask whether the sequence $\left\{x_{n}\right\}$ generated by

$$
x_{n+1}=a_{n} u+b_{n} S_{n} x_{n}+c_{n} T_{n} x_{n}+d_{n} e_{n}
$$

can converge strongly to a point of $\bigcap_{n=1}^{\infty} F\left(S_{n}\right) \cap \bigcap_{n=1}^{\infty} F\left(T_{n}\right)$ provided the coefficient sequences $\left\{a_{n}\right\},\left\{b_{n}\right\},\left\{c_{n}\right\}$ and $\left\{d_{n}\right\}$ are imposed on appropriate conditions, where for any $n \in \mathbb{N}$, each $S_{n}$ is $\mu_{n}$-averaged by $G_{n}$, and each $T_{n}$ is $\lambda_{n}$-averaged by $K_{n}$. We shall show in Section 3 that the sequence $\left\{x_{n}\right\}$ generated by (9) converges strongly to a point of $\bigcap_{n=1}^{\infty} F\left(S_{n}\right) \cap \bigcap_{n=1}^{\infty} F\left(T_{n}\right)$ provided $\bigcap_{n=1}^{\infty} F\left(S_{n}\right) \cap \bigcap_{n=1}^{\infty} F\left(T_{n}\right) \neq \varnothing$ and $\left\{\mu_{n}\right\},\left\{\lambda_{n}\right\}$ and the coefficient sequences $\left\{a_{n}\right\},\left\{b_{n}\right\},\left\{c_{n}\right\}$ and $\left\{d_{n}\right\}$ verify the conditions: 
(i) $\left\{\mu_{n}\right\}$ and $\left\{\lambda_{n}\right\}$ are convergent sequences in $(0,1)$ with limit $\mu, \lambda \in(0,1)$ respectively;

(ii) there are two nonnegative real-valued functions $\kappa_{1}$ and $\kappa_{2}$ on $\mathbb{N}$ with

$$
\begin{aligned}
& \left\|G_{m} x-x\right\|+\left\|K_{m} x-x\right\| \leq \kappa_{1}(m)\left\|G_{n} x-x\right\|+\kappa_{2}(m)\left\|K_{n} x-x\right\|, \\
& \forall m \in \mathbb{N}, \forall n \geq m, \forall x \in C
\end{aligned}
$$

(iii) $\left\{a_{n}\right\},\left\{b_{n}\right\},\left\{c_{n}\right\}$ and $\left\{d_{n}\right\}$ are sequences in $[0,1]$ with $a_{n}+b_{n}+c_{n}+d_{n}=1$ and $a_{n} \in(0,1), \forall n \in \mathbb{N}$;

(iv) $\lim _{n \rightarrow \infty} a_{n}=\lim _{n \rightarrow \infty} \frac{d_{n}}{a_{n}}=0, \sum_{n=1}^{\infty} a_{n}=\infty, \sum_{n=1}^{\infty} d_{n}<\infty$;

(v) $\liminf _{n \rightarrow \infty} b_{n}>0, \liminf _{n \rightarrow \infty} c_{n}>0$.

Based on this main result, we shall deduce many corollaries for averaged mappings in Section 3. Section 4 is devoted to applications. We apply our results in Section 3 to study the split feasibility problem, the zero point problem of maximal monotone operators, the minimization problem and the equilibrium problem, and to show that the unique minimum norm solution can be obtained through our algorithm for each of the aforementioned problems.

\section{Preliminaries}

In order to facilitate our investigation in Section 3, we recall some basic facts. Let $C$ be a nonempty closed convex subset of $\mathcal{H}$. A mapping $T: C \rightarrow \mathcal{H}$ is said to be

(i) nonexpansive if

$$
\|T x-T y\| \leq\|x-y\|, \quad \forall x, y \in C
$$

(ii) firmly nonexpansive if

$$
\|T x-T y\|^{2} \leq\|x-y\|^{2}-\|(I-T) x-(I-T) y\|^{2}, \quad \forall x, y \in C ;
$$

(iii) $\lambda$-averaged by $K$ if

$$
T=(1-\lambda) I+\lambda K
$$

for some $\lambda \in(0,1)$ and some nonexpansive mapping $K$.

If $T: C \rightarrow C$ is nonexpansive, then the fixed point set $F(T)$ of $T$ is closed and convex, cf. [21]. If $T=(1-\lambda) I+\lambda K$ is averaged, then $T$ is nonexpansive with $F(T)=F(K)$.

The metric projection $P_{C}$ from $\mathcal{H}$ onto $C$ is the mapping that assigns each $x \in \mathcal{H}$ the unique point $P_{C} x$ in $C$ with the property

$$
\left\|x-P_{C} x\right\|=\min _{y \in C}\|y-x\|
$$

It is known that $P_{C}$ is nonexpansive and characterized by the inequality: for any $x \in \mathcal{H}$,

$$
\left\langle x-P_{C} x, y-P_{C} x\right\rangle \leq 0, \quad \forall y \in C .
$$

For $\alpha>0$, the resolvent $J_{\alpha}^{A}$ of maximal monotone operator $A$ on $\mathcal{H}$ has the following properties. 
Lemma 2.1 Let A be a maximal monotone operator on $\mathcal{H}$. Then

(a) $J_{\alpha}^{A}$ is single-valued and firmly nonexpansive;

(b) $\mathcal{D}\left(J_{\alpha}^{A}\right)=\mathcal{H}$ and $F\left(J_{\alpha}^{A}\right)=A^{-1} 0$;

(c) (The resolvent identity) for $\mu, \lambda>0$, the following identity holds:

$$
J_{\mu}^{A} x=J_{\lambda}^{A}\left(\frac{\lambda}{\mu} x+\left(1-\frac{\lambda}{\mu}\right) J_{\mu}^{A} x\right), \quad \forall x \in \mathcal{H}
$$

We still need some lemmas that will be quoted in the sequel.

Lemma 2.2 Let $x, y, z \in \mathcal{H}$. Then

(a) $\|x+y\|^{2} \leq\|x\|^{2}+2\langle y, x+y\rangle$;

(b) for any $\lambda \in \mathbb{R}$,

$$
\|\lambda x+(1-\lambda) y\|^{2}=\lambda\|x\|^{2}+(1-\lambda)\|y\|^{2}-\lambda(1-\lambda)\|x-y\|^{2} ;
$$

(c) for $a, b, c \in[0,1]$ with $a+b+c=1$,

$$
\|a x+b y+c z\|^{2}=a\|x\|^{2}+b\|y\|^{2}+c\|z\|^{2}-a b\|x-y\|^{2}-a c\|x-z\|^{2}-b c\|y-z\|^{2} .
$$

Lemma 2.3 (Demiclosedness principle [21]) Let $T$ be a nonexpansive self-mapping on a nonempty closed convex subset $C$ of $\mathcal{H}$, and suppose that $\left\{x_{n}\right\}$ is a sequence in $C$ such that $\left\{x_{n}\right\}$ converges weakly to some $z \in C$ and $\lim _{n \rightarrow \infty}\left\|x_{n}-T x_{n}\right\|=0$. Then $T z=z$.

Lemma 2.4 [18] Let $\left\{s_{n}\right\}$ be a sequence of nonnegative real numbers satisfying

$$
s_{n+1} \leq\left(1-\alpha_{n}\right) s_{n}+\alpha_{n} \mu_{n}+v_{n}, \quad n \in \mathbb{N},
$$

where $\left\{\alpha_{n}\right\},\left\{\mu_{n}\right\}$ and $\left\{v_{n}\right\}$ verify the conditions:

(i) $\left\{\alpha_{n}\right\} \subseteq[0,1], \sum_{n=1}^{\infty} \alpha_{n}=\infty$;

(ii) $\lim \sup _{n \rightarrow \infty} \mu_{n} \leq 0$;

(iii) $\left\{v_{n}\right\} \subseteq[0, \infty)$ and $\sum_{n=1}^{\infty} v_{n}<\infty$.

Then $\lim _{n \rightarrow \infty} s_{n}=0$.

Lemma $2.5[22]$ Let $\left\{s_{n}\right\}$ be a sequence in $\mathbb{R}$ that does not decrease at infinity in the sense that there exists a subsequence $\left\{s_{n_{i}}\right\}$ such that

$$
s_{n_{i}}<s_{n_{i}+1}, \quad \forall i \in \mathbb{N}
$$

For any $k \in \mathbb{N}$, define $m_{k}=\max \left\{j \leq k: s_{j}<s_{j+1}\right\}$. Then $m_{k} \rightarrow \infty$ as $k \rightarrow \infty$ and $\max \left\{s_{m_{k}}, s_{k}\right\} \leq s_{m_{k}+1}, \forall k \in \mathbb{N}$.

\section{Strong convergence theorems}

To establish a strong convergence theorem for averaged mappings $S_{n}, T_{n}, n \in \mathbb{N}$, on $\mathcal{H}$ associated with algorithm (9), we at first need a lemma. 
Lemma 3.1 If $T=(1-\lambda) I+\lambda K$ is a $\lambda$-averaged self-mapping by $K$ on a nonempty closed convex subset $C$ of $\mathcal{H}$ and $p \in F(T)$, then for any $x \in C$, one has

$$
\|T x-p\|^{2} \leq\|x-p\|^{2}-\lambda(1-\lambda)\|x-K x\|^{2} .
$$

Proof Let $x$ be any point in $C$. Then, using $T p=K p=p$ and the nonexpansiveness of $K$, we have from Lemma 2.2(b) that

$$
\begin{aligned}
\|T x-p\|^{2} & =\|T x-T p\|^{2} \\
& =\|(1-\lambda) x+\lambda K x-((1-\lambda) p+K p)\|^{2} \\
& =\|(1-\lambda)(x-p)+\lambda(K x-K p)\|^{2} \\
& =(1-\lambda)\|x-p\|^{2}+\lambda\|K x-K p\|^{2}-\lambda(1-\lambda)\|x-p-(K x-K p)\|^{2} \\
& \leq(1-\lambda)\|x-p\|^{2}+\lambda\|x-p\|^{2}-\lambda(1-\lambda)\|x-K x\|^{2} \\
& =\|x-p\|^{2}-\lambda(1-\lambda)\|x-K x\|^{2} .
\end{aligned}
$$

Theorem 3.2 For any $n \in \mathbb{N}$, suppose that $S_{n}=\left(1-\mu_{n}\right) I+\mu_{n} G_{n}$ and $T_{n}=\left(1-\lambda_{n}\right) I+$ $\lambda_{n} K_{n}$ are averaged self-mappings on a nonempty closed convex subset $C$ of $\mathcal{H}$ with $\Omega:=$ $\bigcap_{n=1}^{\infty} F\left(S_{n}\right) \cap \bigcap_{n=1}^{\infty} F\left(T_{n}\right) \neq \varnothing$, satisfying that

$$
\lim _{n \rightarrow \infty} \mu_{n}=\mu \in(0,1), \quad \lim _{n \rightarrow \infty} \lambda_{n}=\lambda \in(0,1)
$$

and there are two nonnegative real-valued functions $\kappa_{1}$ and $\kappa_{2}$ on $\mathbb{N}$ with

$$
\begin{gathered}
\left\|G_{m} x-x\right\|+\left\|K_{m} x-x\right\| \leq \kappa_{1}(m)\left\|G_{n} x-x\right\|+\kappa_{2}(m)\left\|K_{n} x-x\right\|, \\
\forall m \in \mathbb{N}, \forall n \geq m, \forall x \in C .
\end{gathered}
$$

Suppose further that $\left\{a_{n}\right\},\left\{b_{n}\right\},\left\{c_{n}\right\}$ and $\left\{d_{n}\right\}$ are sequences in $[0,1]$ with $a_{n}+b_{n}+c_{n}+d_{n}=1$ and $a_{n} \in(0,1)$ for all $n \in \mathbb{N}$, and that $\left\{e_{n}\right\}$ and $\left\{v_{n}\right\}$ are two bounded sequences in C. For an arbitrary norm convergent sequence $\left\{u_{n}\right\}$ in $C$ with limit $u$, start with an arbitrary $x_{1}=$ $y_{1} \in C$ and define two sequences $\left\{x_{n}\right\}$ and $\left\{y_{n}\right\}$ by

$$
\begin{aligned}
& x_{n+1}=a_{n} u+b_{n} S_{n} x_{n}+c_{n} T_{n} x_{n}+d_{n} e_{n} ; \\
& y_{n+1}=a_{n} u_{n}+b_{n} S_{n} y_{n}+c_{n} T_{n} y_{n}+d_{n} v_{n} .
\end{aligned}
$$

Then both of $\left\{x_{n}\right\}$ and $\left\{y_{n}\right\}$ converge strongly to $P_{\Omega} u$ provided the following conditions are satisfied:

(i) $\quad \lim _{n \rightarrow \infty} a_{n}=\lim _{n \rightarrow \infty} \frac{d_{n}}{a_{n}}=0, \quad \sum_{n=1}^{\infty} a_{n}=\infty, \quad \sum_{n=1}^{\infty} d_{n}<\infty$;

(ii) $\quad \liminf _{n \rightarrow \infty} b_{n}>0, \quad \liminf _{n \rightarrow \infty} c_{n}>0$.

Moreover, when every $S_{n}$ is the identity mapping I, the result still holds without the condition $\liminf _{n \rightarrow \infty} b_{n}>0$. 
Proof Put $p=P_{\Omega} u$. Firstly, we show that $\left\{x_{n}\right\}$ converges strongly to $p$. It comes from the nonexpansiveness of $S_{n}$ and $T_{n}$ that

$$
\begin{aligned}
\left\|x_{n+1}-p\right\| & =\left\|a_{n}(u-p)+b_{n}\left(S_{n} x_{n}-p\right)+c_{n}\left(T_{n} x_{n}-p\right)+d_{n}\left(e_{n}-p\right)\right\| \\
& =a_{n}\|u-p\|+\left(b_{n}+c_{n}\right)\left\|x_{n}-p\right\|+d_{n}\left\|e_{n}-p\right\|,
\end{aligned}
$$

from which it follows that $\left\{x_{n}\right\}$ is a bounded sequence. Taking into account of Lemma 2.2 and using Lemma 3.1, we get

$$
\begin{aligned}
&\left\|x_{n+1}-p\right\|^{2} \\
&=\left\|a_{n}(u-p)+b_{n}\left(S_{n} x_{n}-p\right)+c_{n}\left(T_{n} x_{n}-p\right)+d\left(e_{n}-p\right)\right\|^{2} \\
& \leq\left\|b_{n}\left(S_{n} x_{n}-p\right)+c_{n}\left(T_{n} x_{n}-p\right)+d_{n}\left(e_{n}-p\right)\right\|^{2}+2 a_{n}\left\langle u-p, x_{n+1}-p\right\rangle \\
&=\left(1-a_{n}\right)^{2}\left\|\frac{b_{n}}{1-a_{n}}\left(S_{n} x_{n}-p\right)+\frac{c_{n}}{1-a_{n}}\left(T_{n} x_{n}-p\right)+\frac{d_{n}}{1-a_{n}}\left(e_{n}-p\right)\right\|^{2} \\
&+2 a_{n}\left\langle u-p, x_{n+1}-p\right\rangle \\
& \leq\left(1-a_{n}\right)^{2}\left(\frac{b_{n}}{1-a_{n}}\left\|S_{n} x_{n}-p\right\|^{2}+\frac{c_{n}}{1-a_{n}}\left\|T_{n} x_{n}-p\right\|^{2}+\frac{d_{n}}{1-a_{n}}\left\|e_{n}-p\right\|^{2}\right) \\
&+2 a_{n}\left\langle u-p, x_{n+1}-p\right\rangle \\
& \leq b_{n}\left\|S_{n} x_{n}-p\right\|^{2}+c_{n}\left\|T_{n} x_{n}-p\right\|^{2}+d_{n}\left\|e_{n}-p\right\|^{2}+2 a_{n}\left\langle u-p, x_{n+1}-p\right\rangle \\
& \leq b_{n}\left(\left\|x_{n}-p\right\|^{2}-\mu_{n}\left(1-\mu_{n}\right)\left\|x_{n}-G_{n} x_{n}\right\|^{2}\right) \\
&+c_{n}\left(\left\|x_{n}-p\right\|^{2}-\lambda_{n}\left(1-\lambda_{n}\right)\left\|x_{n}-K_{n} x_{n}\right\|^{2}\right)+d_{n}\left\|e_{n}-p\right\|^{2}+2 a_{n}\left\langle u-p, x_{n+1}-p\right\rangle \\
&=\left(b_{n}+c_{n}\right)\left\|x_{n}-p\right\|^{2}+d_{n}\left\|e_{n}-p\right\|^{2}+2 a_{n}\left\langle u-p, x_{n+1}-p\right\rangle \\
&-b_{n} \mu_{n}\left(1-\mu_{n}\right)\left\|x_{n}-G_{n} x_{n}\right\|^{2}-c_{n} \lambda_{n}\left(1-\lambda_{n}\right)\left\|x_{n}-K_{n} x_{n}\right\|^{2} .
\end{aligned}
$$

We now carry on with the proof by considering the following two cases: (I) $\left\{\left\|x_{n}-p\right\|\right\}$ is eventually decreasing, and (II) $\left\{\left\|x_{n}-p\right\|\right\}$ is not eventually decreasing.

Case I: Suppose that $\left\{\left\|x_{n}-p\right\|\right\}$ is eventually decreasing, that is, there is $N \in \mathbb{N}$ such that $\left\{\left\|x_{n}-p\right\|\right\}_{n \geq N}$ is decreasing. In this case, $\lim _{n \rightarrow \infty}\left\|x_{n}-p\right\|$ exists in $\mathbb{R}$. By condition (ii), we may assume that there are two $b, c \in(0,1)$ such that $b \leq b_{n}$ and $c \leq c_{n}$ for all $n \in \mathbb{N}$. Then from inequality (11) we have

$$
\begin{aligned}
0 & \leq b \mu_{n}\left(1-\mu_{n}\right)\left\|x_{n}-G_{n} x_{n}\right\|^{2}+c \lambda_{n}\left(1-\lambda_{n}\right)\left\|x_{n}-K_{n} x_{n}\right\|^{2} \\
& \leq b_{n} \mu_{n}\left(1-\mu_{n}\right)\left\|x_{n}-G_{n} x_{n}\right\|^{2}+c_{n} \lambda_{n}\left(1-\lambda_{n}\right)\left\|x_{n}-K_{n} x_{n}\right\|^{2} \\
& \leq\left(b_{n}+c_{n}\right)\left\|x_{n}-p\right\|^{2}-\left\|x_{n+1}-p\right\|^{2}+d_{n}\left\|e_{n}-p\right\|^{2}+2 a_{n}\left\langle u-p, x_{n+1}-p\right\rangle \\
& =\left(1-\left(a_{n}+d_{n}\right)\right)\left\|x_{n}-p\right\|^{2}-\left\|x_{n+1}-p\right\|^{2}+d_{n}\left\|e_{n}-p\right\|^{2}+2 a_{n}\left\langle u-p, x_{n+1}-p\right\rangle
\end{aligned}
$$

and noting via condition (i) that

$$
\begin{aligned}
& \lim _{n \rightarrow \infty}\left(1-\left(a_{n}+d_{n}\right)\right)\left\|x_{n}-p\right\|^{2}=\lim _{n \rightarrow \infty}\left\|x_{n+1}-p\right\|^{2} \text { and } \\
& \lim _{n \rightarrow \infty} d_{n}\left\|e_{n}-p\right\|^{2}=\lim _{n \rightarrow \infty} 2 a_{n}\left\langle u-p, x_{n+1}-p\right\rangle=0,
\end{aligned}
$$


we conclude that

$$
\lim _{n \rightarrow \infty}\left(b \mu_{n}\left(1-\mu_{n}\right)\left\|x_{n}-G_{n} x_{n}\right\|^{2}+c \lambda_{n}\left(1-\lambda_{n}\right)\left\|x_{n}-K_{n} x_{n}\right\|^{2}\right)=0,
$$

which implies that

$$
\lim _{n \rightarrow \infty}\left\|x_{n}-G_{n} x_{n}\right\|=\lim _{n \rightarrow \infty}\left\|x_{n}-K_{n} x_{n}\right\|=0 .
$$

Then from condition (3.2) we deduce for all $m \in \mathbb{N}$ that

$$
\lim _{n \rightarrow \infty}\left\|x_{n}-G_{m} x_{n}\right\|=\lim _{n \rightarrow \infty}\left\|x_{n}-K_{m} x_{n}\right\|=0 .
$$

Since $\left\{x_{n}\right\}$ is bounded, it has a subsequence $\left\{x_{n_{k}}\right\}$ such that $\left\{x_{n_{k}}\right\}$ converges weakly to some $z \in \mathcal{H}$ and

$$
\limsup _{n \rightarrow \infty}\left\langle u-p, x_{n+1}-p\right\rangle=\lim _{k \rightarrow \infty}\left\langle u-p, x_{n_{k}}-p\right\rangle=\langle u-p, z-p\rangle \leq 0,
$$

where the last inequality follows from (13) since $z \in \Omega$ by Lemma 2.3. Choose $M>0$ so that $\sup \left\{\left\|e_{n}-p\right\|^{2}+2\|u-p\|\left\|x_{n+1}-p\right\|: n \in \mathbb{N}\right\} \leq M$. From (11) we have

$$
\begin{aligned}
&\left\|x_{n+1}-p\right\|^{2} \\
& \leq\left(1-\left(a_{n}+d_{n}\right)\right)\left\|x_{n}-p\right\|^{2}+\left(a_{n}+d_{n}\right) \cdot 2\left\langle u-p, x_{n+1}-p\right\rangle \\
& \quad \quad d_{n}\left(\left\|e_{n}-p\right\|^{2}+2\|u-p\|\left\|x_{n+1}-p\right\|\right) \\
& \leq\left(1-\left(a_{n}+d_{n}\right)\right)\left\|x_{n}-p\right\|^{2}+\left(a_{n}+d_{n}\right) \cdot 2\left\langle u-p, x_{n+1}-p\right\rangle+d_{n} M .
\end{aligned}
$$

Accordingly, because of (14) and condition (i), we can apply Lemma 2.4 to inequality (15) with $s_{n}=\left\|x_{n}-p\right\|^{2}, \alpha_{n}=a_{n}+d_{n}, \mu_{n}=2\left\langle u-p, x_{n+1}-p\right\rangle$ and $v_{n}=d_{n} M$ to conclude that

$$
\lim _{n \rightarrow \infty} x_{n}=p
$$

Case II: Suppose that $\left\{\left\|x_{n}-p\right\|\right\}$ is not eventually decreasing. In this case, by Lemma 2.5, there exists a nondecreasing sequence $\left\{m_{k}\right\}$ in $\mathbb{N}$ such that $m_{k} \rightarrow \infty$ and

$$
\max \left\{\left\|x_{m_{k}}-p\right\|,\left\|x_{k}-p\right\|\right\} \leq\left\|x_{m_{k}+1}-p\right\|, \quad \forall k \in \mathbb{N} .
$$

Then it follows from (11) and (16) that

$$
\begin{aligned}
\| x_{m_{k}} & -p \|^{2} \\
\leq & \left\|x_{m_{k}+1}-p\right\|^{2} \\
\leq & \left(b_{m_{k}}+c_{m_{k}}\right)\left\|x_{m_{k}}-p\right\|^{2}+d_{m_{k}}\left\|e_{m_{k}}-p\right\|^{2} \\
& +2 a_{m_{k}}\left\langle u-p, x_{m_{k}+1}-p\right\rangle-b_{m_{k}} \mu_{m_{k}}\left(1-\mu_{m_{k}}\right)\left\|x_{m_{k}}-G_{m_{k}} x_{m_{k}}\right\|^{2} \\
& \quad-c_{m_{k}} \lambda_{m_{k}}\left(1-\lambda_{m_{k}}\right)\left\|x_{m_{k}}-K_{m_{k}} x_{m_{k}}\right\|^{2} .
\end{aligned}
$$


Therefore,

$$
\begin{aligned}
0 & \leq b_{m_{k}} \mu_{m_{k}}\left(1-\mu_{m_{k}}\right)\left\|x_{m_{k}}-G_{m_{k}} x_{m_{k}}\right\|^{2}+c_{m_{k}} \lambda_{m_{k}}\left(1-\lambda_{m_{k}}\right)\left\|x_{m_{k}}-K_{m_{k}} x_{m_{k}}\right\|^{2} \\
& \leq-\left(1-\left(b_{m_{k}}+c_{m_{k}}\right)\right)\left\|x_{m_{k}}-p\right\|^{2}+d_{m_{k}}\left\|e_{m_{k}}-p\right\|^{2}+2 a_{m_{k}}\left\langle u-p, x_{m_{k}+1}-p\right\rangle \\
& =-\left(a_{m_{k}}+d_{m_{k}}\right)\left\|x_{m_{k}}-p\right\|^{2}+d_{m_{k}}\left\|e_{m_{k}}-p\right\|^{2}+2 a_{m_{k}}\left\langle u-p, x_{m_{k}+1}-p\right\rangle,
\end{aligned}
$$

and then proceeding just as in the proof in Case I, we obtain

$$
\lim _{k \rightarrow \infty}\left\|x_{m_{k}}-G_{m_{k}} x_{m_{k}}\right\|=\lim _{k \rightarrow \infty}\left\|x_{m_{k}}-K_{m_{k}} x_{m_{k}}\right\|=0
$$

which in conjunction with condition (3.2) shows for all $m_{j}$ that

$$
\lim _{k \rightarrow \infty}\left\|x_{m_{k}}-G_{m_{j}} x_{m_{k}}\right\|=\lim _{k \rightarrow \infty}\left\|x_{m_{k}}-K_{m_{j}} x_{m_{k}}\right\|=0,
$$

and then it follows that

$$
\limsup _{k \rightarrow \infty}\left\langle u-p, x_{m_{k}+1}-p\right\rangle \leq 0
$$

From (17) we have

$$
\left(1-\left(b_{m_{k}}+c_{m_{k}}\right)\right)\left\|x_{m_{k}}-p\right\|^{2} \leq d_{m_{k}}\left\|e_{m_{k}}-p\right\|^{2}+2 a_{m_{k}}\left\langle u-p, x_{m_{k}+1}-p\right\rangle,
$$

and thus

$$
\begin{aligned}
\left\|x_{m_{k}}-p\right\|^{2} & \leq \frac{d_{m_{k}}}{a_{m_{k}}+d_{m_{k}}}\left\|e_{m_{k}}-p\right\|^{2}+\frac{2 a_{m_{k}}}{a_{m_{k}}+d_{m_{k}}}\left\langle u-p, x_{m_{k}+1}-p\right\rangle \\
& \leq \frac{d_{m_{k}}}{a_{m_{k}}}\left\|e_{m_{k}}-p\right\|^{2}+2\left\langle u-p, x_{m_{k}+1}-p\right\rangle .
\end{aligned}
$$

Letting $k \rightarrow \infty$ and using (19) and condition (i), we obtain

$$
\lim _{k \rightarrow \infty}\left\|x_{m_{k}}-p\right\|=0
$$

Also, since

$$
\begin{aligned}
& \left\|x_{m_{k}+1}-x_{m_{k}}\right\| \\
& \quad \leq a_{m_{k}}\left\|u-x_{m_{k}}\right\|+b_{m_{k}} \mu_{m_{k}}\left\|G_{m_{k}} x_{m_{k}}-x_{m_{k}}\right\|+c_{m_{k}} \lambda_{m_{k}}\left\|K_{m_{k}} x_{m_{k}}-x_{m_{k}}\right\| \\
& \quad+d_{m_{k}}\left\|e_{m_{k}}-x_{m_{k}}\right\|,
\end{aligned}
$$

which together with (18) implies $\lim _{k \rightarrow \infty}\left\|x_{m_{k}+1}-x_{m_{k}}\right\|=0$, and so

$$
\lim _{k \rightarrow \infty}\left\|x_{m_{k}+1}-p\right\|=0
$$

by virtue of (20). Consequently, we conclude $\lim _{k \rightarrow \infty}\left\|x_{k}-p\right\|=0$ via (16) and (21). In addition, note that the condition $\liminf _{n \rightarrow \infty} b_{n}=0$ is used to establish $\lim _{n \rightarrow \infty}\left\|x_{n}-G_{n} x_{n}\right\|=0$ 
and $\lim _{k \rightarrow \infty}\left\|x_{m_{k}}-G_{m_{k}} x_{m_{k}}\right\|=0$ in (12) and (18) respectively. However, both limits hold trivially without this condition provided every $S_{n}$ is the identity mapping $I$.

Next, we show that $\left\{y_{n}\right\}$ converges strongly to $p$ too. Applying Lemma 2.4 to the following inequality

$$
\begin{aligned}
\left\|y_{n+1}-x_{n+1}\right\| & \leq a_{n}\left\|u_{n}-u\right\|+\left(b_{n}+c_{n}\right)\left\|y_{n}-x_{n}\right\|+d_{n}\left\|v_{n}-e_{n}\right\| \\
& =\left(1-\left(a_{n}+d_{n}\right)\right)\left\|y_{n}-x_{n}\right\|+a_{n}\left\|u_{n}-u\right\|+d_{n}\left\|v_{n}-e_{n}\right\|
\end{aligned}
$$

for all $n \in \mathbb{N}$, we see that $\lim _{n \rightarrow \infty}\left\|y_{n}-x_{n}\right\|=0$, and hence $\lim _{n \rightarrow \infty} y_{n}=p$ follows. This completes the proof.

The following lemma is easily proved and so its proof is omitted.

Lemma 3.3 For any $n \in \mathbb{N}$, suppose that $S_{n}=\left(1-\mu_{n}\right) I+\mu_{n} G_{n}$ and $T_{n}=\left(1-\lambda_{n}\right) I+\lambda_{n} K_{n}$ are averaged self-mappings on a nonempty closed convex subset $C$ of $\mathcal{H}$ such that condition (3.1) holds. Then $\left\{S_{n}\right\}$ and $\left\{T_{n}\right\}$ satisfy condition (3.2) if and only if $\left\{G_{n}\right\}$ and $\left\{K_{n}\right\}$ satisfy condition (3.2).

If the sequence $\left\{S_{n}\right\}$ (resp. $\left\{T_{n}\right\}$ ) of averaged mappings consists of a single mapping $S$ (resp. $T$ ), then $\left\{S_{n}\right\}$ and $\left\{T_{n}\right\}$ obviously verify conditions (3.1) and (3.2), and hence from Lemma 3.3 we have the following corollary.

Corollary 3.4 Suppose $S$ and $T$ are two averaged self-mappings on a nonempty closed convex subset $C$ of $\mathcal{H}$ with $\Omega=F(S) \cap F(T) \neq \varnothing$, and suppose that $\left\{a_{n}\right\},\left\{b_{n}\right\},\left\{c_{n}\right\}$ and $\left\{d_{n}\right\}$ are sequences in $[0,1]$ with $a_{n}+b_{n}+c_{n}+d_{n}=1$ and $a_{n} \in(0,1)$ for all $n \in \mathbb{N}$, and $\left\{e_{n}\right\}$ and $\left\{v_{n}\right\}$ are two bounded sequences in $C$. For an arbitrary norm convergent sequence $\left\{u_{n}\right\}$ in $C$ with limit $u$, start with an arbitrary $x_{1}=y_{1} \in C$ and define two sequences $\left\{x_{n}\right\}$ and $\left\{y_{n}\right\}$ by

$$
\begin{aligned}
& x_{n+1}=a_{n} u+b_{n} S x_{n}+c_{n} T x_{n}+d_{n} e_{n} ; \\
& y_{n+1}=a_{n} u_{n}+b_{n} S y_{n}+c_{n} T y_{n}+d_{n} v_{n} .
\end{aligned}
$$

Then both of $\left\{x_{n}\right\}$ and $\left\{y_{n}\right\}$ converge strongly to $P_{\Omega} u$ provided the following conditions are satisfied:

(i) $\quad \lim _{n \rightarrow \infty} a_{n}=\lim _{n \rightarrow \infty} \frac{d_{n}}{a_{n}}=0, \quad \sum_{n=1}^{\infty} a_{n}=\infty, \quad \sum_{n=1}^{\infty} d_{n}<\infty$;

(ii) $\quad \liminf _{n \rightarrow \infty} b_{n}>0, \quad \liminf _{n \rightarrow \infty} c_{n}>0$.

Moreover, when $S$ is the identity mapping $I$, the result still holds without the condition $\liminf _{n \rightarrow \infty} b_{n}>0$.

Theorem 3.5 For any $n \in \mathbb{N}$, suppose $S_{n}$ and $T_{n}$ are firmly nonexpansive self-mappings on a nonempty closed convex subset $C$ of $\mathcal{H}$ with $\Omega:=\bigcap_{n=1}^{\infty} F\left(S_{n}\right) \cap \bigcap_{n=1}^{\infty} F\left(T_{n}\right) \neq \varnothing$, satisfying condition (3.2). Suppose further that $\left\{a_{n}\right\},\left\{b_{n}\right\},\left\{c_{n}\right\}$ and $\left\{d_{n}\right\}$ are sequences in $[0,1]$ with 
$a_{n}+b_{n}+c_{n}+d_{n}=1$ and $a_{n} \in(0,1)$ for all $n \in \mathbb{N}$, and $\left\{e_{n}\right\}$ and $\left\{v_{n}\right\}$ are two bounded sequences in $C$. For an arbitrary norm convergent sequence $\left\{u_{n}\right\}$ in $C$ with limit $u$, start with an arbitrary $x_{1}=y_{1} \in C$ and define two sequences $\left\{x_{n}\right\}$ and $\left\{y_{n}\right\}$ by

$$
\begin{aligned}
& x_{n+1}=a_{n} u+b_{n} S_{n} x_{n}+c_{n} T_{n} x_{n}+d_{n} e_{n} \\
& y_{n+1}=a_{n} u_{n}+b_{n} S_{n} y_{n}+c_{n} T_{n} y_{n}+d_{n} v_{n} .
\end{aligned}
$$

Then both of $\left\{x_{n}\right\}$ and $\left\{y_{n}\right\}$ converge strongly to $P_{\Omega} u$ provided the following conditions are satisfied:

(i) $\quad \lim _{n \rightarrow \infty} a_{n}=\lim _{n \rightarrow \infty} \frac{d_{n}}{a_{n}}=0, \quad \sum_{n=1}^{\infty} a_{n}=\infty, \quad \sum_{n=1}^{\infty} d_{n}<\infty$;

(ii) $\quad \liminf _{n \rightarrow \infty} b_{n}>0, \quad \liminf _{n \rightarrow \infty} c_{n}>0$.

Moreover, when every $S_{n}$ is the identity mapping I, the result still holds without the condition $\liminf _{n \rightarrow \infty} b_{n}>0$.

Proof Since any firmly nonexpansive mapping is $\frac{1}{2}$-averaged, condition (3.1) holds, and hence by Lemma 3.3 we see that all the requirements of Theorem 3.2 are verified. Therefore, the desired conclusion follows.

If $S_{n}=I$ and $d_{n}=0$ for all $n \in \mathbb{N}$ in Theorem 3.2, then we have the following corollary.

Corollary 3.6 Suppose, for all $n \in \mathbb{N}$, that $T_{n}=\left(1-\lambda_{n}\right) I+\lambda_{n} K_{n}$ is an averaged self-mapping on a nonempty closed convex subset $C$ of $\mathcal{H}$ with $\Omega=\bigcap_{n=1}^{\infty} F\left(T_{n}\right) \neq \varnothing$, and $\lim _{n \rightarrow \infty} \lambda_{n}=\lambda \in$ $(0,1)$, and assume that condition (3.2) holds for $\{I\}$ and $\left\{T_{n}\right\}$. Suppose further that $\left\{a_{n}\right\}$, $\left\{b_{n}\right\}$ and $\left\{c_{n}\right\}$ are sequences in $[0,1]$ with $a_{n}+b_{n}+c_{n}=1$ and $a_{n} \in(0,1)$ for all $n \in \mathbb{N}$. Let $\left\{\alpha_{n}\right\}$ be a sequence in $(0, \infty)$. For an arbitrary fixed $u \in C$, start with an arbitrary $x_{1} \in C$ and define

$$
x_{n+1}=a_{n} u+b_{n} x_{n}+c_{n} T_{n} x_{n}, \quad n \in \mathbb{N} \text {. }
$$

Then the sequence $\left\{x_{n}\right\}$ converges strongly to $P_{\Omega} u$ provided the following conditions are satisfied:

$$
\lim _{n \rightarrow \infty} a_{n}=0, \quad \sum_{n=1}^{\infty} a_{n}=\infty, \quad \liminf _{n \rightarrow \infty} c_{n}>0 .
$$

Corollary 3.7 Suppose, for all $n \in \mathbb{N}$, that $T_{n}=\left(1-\lambda_{n}\right) I+\lambda_{n} K_{n}$ is an averaged self-mapping on $\mathcal{H}$ with $\Omega=\bigcap_{n=1}^{\infty} F\left(T_{n}\right) \neq \varnothing$, and $\lim _{n \rightarrow \infty} \lambda_{n}=\lambda \in(0,1)$, and assume that condition (3.2) holds for $\{I\}$ and $\left\{T_{n}\right\}$. Suppose further that $\left\{a_{n}\right\},\left\{b_{n}\right\}$ and $\left\{c_{n}\right\}$ are sequences in $[0,1]$ with $a_{n}+b_{n}+c_{n}=1$ and $a_{n} \in(0,1)$ for all $n \in \mathbb{N}$, and that $\left\{e_{n}\right\}$ is a bounded sequence in $\mathcal{H}$. Let $\left\{\alpha_{n}\right\}$ be a sequence in $(0, \infty)$. For an arbitrary fixed $u \in \mathcal{H}$, start with an arbitrary $x_{1} \in \mathcal{H}$ and define

$$
x_{n+1}=a_{n} u+b_{n} x_{n}+c_{n} T_{n} x_{n}+e_{n}, \quad n \in \mathbb{N} \text {. }
$$


Then the sequence $\left\{x_{n}\right\}$ converges strongly to $P_{\Omega} u$ provided the following conditions are satisfied:

(i) $\quad \lim _{n \rightarrow \infty} a_{n}=0, \quad \sum_{n=1}^{\infty} a_{n}=\infty, \quad \liminf _{n \rightarrow \infty} c_{n}>0$;

(ii) either $\lim _{n \rightarrow \infty} \frac{\left\|e_{n}\right\|}{a_{n}}=0$ or $\sum_{n=1}^{\infty}\left\|e_{n}\right\|<\infty$.

Proof Put $p=P_{\Omega} u$. Let $z_{1}=x_{1}$ and define a sequence $\left\{z_{n}\right\}$ iteratively by

$$
z_{n+1}=a_{n} u+b_{n} z_{n}+c_{n} T_{n} z_{n} .
$$

We have $\lim _{n \rightarrow \infty} z_{n}=p$ by Corollary 3.6. Since

$$
\begin{aligned}
\left\|x_{n+1}-z_{n+1}\right\| & \leq b_{n}\left\|x_{n}-z_{n}\right\|+c_{n}\left\|T_{n} x_{n}-T_{n} z_{n}\right\|+\left\|e_{n}\right\| \\
& \leq\left(b_{n}+c_{n}\right)\left\|x_{n}-z_{n}\right\|+\left\|e_{n}\right\| \\
& =\left(1-a_{n}\right)\left\|x_{n}-z_{n}\right\|+\left\|e_{n}\right\|,
\end{aligned}
$$

the limit $\lim _{n \rightarrow \infty}\left\|x_{n}-z_{n}\right\|=0$ follows by applying Lemma 2.4 to (22), and thus,

$$
\lim _{n \rightarrow \infty} x_{n}=p
$$

\section{Applications}

In this section, we shall apply some of the strong convergence theorems in Section 3 to approximate a solution of the split feasibility problem, a common zero of maximal monotone operators, a minimizer of a proper lower semicontinuous convex function, and to study the related equilibrium problem.

$\mathrm{Xu}$ [2] transformed SFP (1) to the fixed point problem of the operator (3):

$$
P_{C}\left(I-\gamma A^{*}\left(I-P_{Q}\right) A\right)
$$

He proved Lemma 4.1 below.

Lemma 4.1 [2] A point $x^{*} \in \mathcal{H}_{1}$ solves SFP(1) if and only if $x^{*}$ is a fixed point of the operator (3): $P_{C}\left(I-\gamma A^{*}\left(I-P_{Q}\right) A\right)$.

Moreover, in the proof of Theorem 3.6 of [2], Xu showed the following lemma.

Lemma 4.2 [2] For any $\gamma \in \mathbb{R}$ with $0<\gamma<\frac{2}{\|A\|^{2}}$, the operator (3): $P_{C}\left(I-\gamma A^{*}\left(I-P_{Q}\right) A\right)$ is $\frac{2+\gamma\|A\|^{2}}{4}$-averaged.

Invoking Lemmas 4.1 and 4.2, we obtain the theorem below from Corollary 3.4 by putting $S=I$ and $T=P_{C}\left(I-\gamma A^{*}\left(I-P_{Q}\right) A\right)$ for all $n \in \mathbb{N}$.

Theorem 4.3 Let $C$ and $Q$ be nonempty closed convex subsets of two Hilbert spaces $\mathcal{H}_{1}$ and $\mathcal{H}_{2}$ respectively, and let $A: \mathcal{H}_{1} \rightarrow \mathcal{H}_{2}$ be a bounded linear mapping. Put $T=P_{C}(I-$ 
$\left.\gamma A^{*}\left(I-P_{Q}\right) A\right)$, where $\gamma$ satisfies $0<\gamma<\frac{2}{\|A\|^{2}}$. Suppose that the solution set $\Omega$ of SFP (1) is nonempty, and suppose further that $\left\{a_{n}\right\},\left\{b_{n}\right\},\left\{c_{n}\right\}$ and $\left\{d_{n}\right\}$ are sequences in $[0,1]$ with $a_{n}+b_{n}+c_{n}+d_{n}=1$ and $a_{n} \in(0,1)$ for all $n \in \mathbb{N}$, and that $\left\{e_{n}\right\}$ is a bounded sequence in $C$. For an arbitrary fixed $u \in C$, start with an arbitrary $x_{1} \in C$ and define the sequence $\left\{x_{n}\right\}$ by

$$
x_{n+1}=a_{n} u+b_{n} x_{n}+c_{n} T x_{n}+d_{n} e_{n} .
$$

Then $\left\{x_{n}\right\}$ converges strongly to $P_{\Omega} u$ provided the following conditions are satisfied:

(i) $\quad \lim _{n \rightarrow \infty} a_{n}=\lim _{n \rightarrow \infty} \frac{d_{n}}{a_{n}}=0, \quad \sum_{n=1}^{\infty} a_{n}=\infty, \quad \sum_{n=1}^{\infty} d_{n}<\infty$;

(ii) $\liminf _{n \rightarrow \infty} c_{n}>0$.

When the point $u$ in the above theorem is taken to be 0 , we see that the limit point $v$ of the sequence $\left\{x_{n}\right\}$ is the unique minimum norm solution of $\operatorname{SFP}(1)$, that is, $\|v\|=\min \{\hat{x}$ : $\hat{x} \in \Omega\}$.

Here, readers may compare the above theorem with Theorem 3.6 of [2], which says, for $0<\gamma<\frac{2}{\|A\|^{2}}$ and sequence $\left\{a_{n}\right\}$ in $\left[0, \frac{4}{2+\gamma\|A\|^{2}}\right]$ satisfying

$$
\sum_{n=1}^{\infty} a_{n}\left(\frac{4}{2+\gamma\|A\|^{2}}-a_{n}\right)=\infty,
$$

that the sequence $\left\{x_{n}\right\}$ generated by

$$
x_{n+1}=\left(1-a_{n}\right) x_{n}+a_{n} P_{C}\left(I-\gamma A^{*}\left(I-P_{Q}\right) A\right) x_{n}
$$

converges weakly to a solution of SFP (1) provided the solution set of SFP (1) is nonempty. It is also interesting to compare Theorem 4.3 with Theorem 5.5 of [2] and Theorem 3.1 of [5]. Our method is different from those in [2] and [5] even in the case of $u=0$, because our algorithm contains an error term and uses the operator $P_{C}\left(I-\gamma A^{*}\left(I-P_{\mathrm{Q}}\right) A\right)$ directly without any regularization.

Theorem 4.4 Let $C$ and $Q$ be nonempty closed convex subsets of two Hilbert spaces $\mathcal{H}_{1}$ and $\mathcal{H}_{2}$ respectively, and let $A, B$ be bounded linear mappings from $\mathcal{H}_{1}$ to $\mathcal{H}_{2}$. Put $S=P_{C}(I-$ $\left.\gamma B^{*}\left(I-P_{Q}\right) B\right)$ and $T=P_{C}\left(I-\gamma A^{*}\left(I-P_{Q}\right) A\right)$, where $\gamma$ satisfies $0<\gamma<\min \left\{\frac{2}{\|B\|^{2}}, \frac{2}{\|A\|^{2}}\right\}$. Suppose the solution set $\Omega$ of SFP (5) is nonempty, and suppose further that $\left\{a_{n}\right\},\left\{b_{n}\right\},\left\{c_{n}\right\}$ and $\left\{d_{n}\right\}$ are sequences in $[0,1]$ with $a_{n}+b_{n}+c_{n}+d_{n}=1$, and $a_{n} \in(0,1)$ for all $n \in \mathbb{N}$, and that $\left\{e_{n}\right\}$ is a bounded sequence in $C$. For an arbitrary fixed $u \in C$, start with an arbitrary $x_{1} \in C$ and define the sequence $\left\{x_{n}\right\}$ by

$$
x_{n+1}=a_{n} u+b_{n} S x_{n}+c_{n} T x_{n}+d_{n} e_{n} .
$$

Then $\left\{x_{n}\right\}$ converges strongly to $P_{\Omega} u$ provided the following conditions are satisfied:

$$
\begin{aligned}
& \text { (i) } \lim _{n \rightarrow \infty} a_{n}=\lim _{n \rightarrow \infty} \frac{d_{n}}{a_{n}}=0, \quad \sum_{n=1}^{\infty} a_{n}=\infty, \quad \sum_{n=1}^{\infty} d_{n}<\infty \text {; } \\
& \text { (ii) } \liminf _{n \rightarrow \infty} b_{n}>0, \quad \liminf _{n \rightarrow \infty} c_{n}>0 .
\end{aligned}
$$


Proof It is clear that this theorem follows from Lemmas 4.1 and 4.2 and Corollary 3.4.

Replacing $S_{n}$ and $T_{n}$ in Theorem 3.2 with the resolvents $J_{\beta_{n}}^{B}$ and $J_{\alpha_{n}}^{A}$ of two maximal monotone operators $B$ and $A$ respectively, we have Theorem 4.5 below.

Theorem 4.5 Suppose that $B$ and $A$ are two maximal monotone operators on $\mathcal{H}$ with $B^{-1} 0 \cap A^{-1} 0 \neq \varnothing$, and suppose that $\left\{a_{n}\right\},\left\{b_{n}\right\},\left\{c_{n}\right\}$ and $\left\{d_{n}\right\}$ are sequences in $[0,1]$ with $a_{n}+b_{n}+c_{n}+d_{n}=1$ and $a_{n} \in(0,1)$ for all $n \in \mathbb{N}$. Let $\left\{\alpha_{n}\right\}$ and $\left\{\beta_{n}\right\}$ be sequences in $(0, \infty)$, and let $\left\{e_{n}\right\}$ and $\left\{v_{n}\right\}$ be two bounded sequences in $\mathcal{H}$. For an arbitrary norm convergent sequence $\left\{u_{n}\right\}$ in $\mathcal{H}$ with limit $u$, start with an arbitrary $x_{1}=y_{1} \in \mathcal{H}$ and define two sequences $\left\{x_{n}\right\}$ and $\left\{y_{n}\right\}$ by

$$
\begin{aligned}
& x_{n+1}=a_{n} u+b_{n} J_{\beta_{n}}^{B} x_{n}+c_{n} J_{\alpha_{n}}^{A} x_{n}+d_{n} e_{n} ; \\
& y_{n+1}=a_{n} u_{n}+b_{n} J_{\beta_{n}}^{B} y_{n}+c_{n} J_{\alpha_{n}}^{A} y_{n}+d_{n} v_{n} .
\end{aligned}
$$

Then both of the sequences $\left\{x_{n}\right\}$ and $\left\{y_{n}\right\}$ converge strongly to $P_{B^{-1} 0 \cap A^{-1} 0} u$ provided the following conditions are satisfied:

(i) $\quad \lim _{n \rightarrow \infty} a_{n}=\lim _{n \rightarrow \infty} \frac{d_{n}}{a_{n}}=0, \quad \sum_{n=1}^{\infty} a_{n}=\infty, \quad \sum_{n=1}^{\infty} d_{n}<\infty$;

(ii) $\quad \liminf _{n \rightarrow \infty} b_{n}>0, \quad \liminf _{n \rightarrow \infty} c_{n}>0$;

(iii) $\quad \liminf _{n \rightarrow \infty} \alpha_{n}>0, \quad \liminf _{n \rightarrow \infty} \beta_{n}>0$.

Proof Since all the requirements of Theorem 3.2 are satisfied except conditions (3.1) and (3.2), we have to check these two conditions. For any $n \in \mathbb{N}$, let $S_{n}=J_{\beta_{n}}^{B}$ and $T_{n}=J_{\alpha_{n}}^{A}$. By Lemma 2.1(b), we have $B^{-1} 0=F\left(S_{n}\right)$ and $A^{-1} 0=F\left(T_{n}\right)$ for all $n \in \mathbb{N}$. Moreover, since all $S_{n}$ and $T_{n}$ are firmly nonexpansive, all of them are $\frac{1}{2}$-averaged, so condition (3.1) is satisfied with $\mu_{n}=\lambda_{n}=\frac{1}{2}$ for all $n \in \mathbb{N}$. According to Lemma 3.3, it remains to prove that condition (3.2) holds for $\left\{J_{\beta_{n}}^{B}\right\}$ and $\left\{J_{\alpha_{n}}^{A}\right\}$. Since condition (iii) holds, we may assume that there is $\tau \in(0,1)$ such that $\tau<\alpha_{n}$ and $\tau<\beta_{n}$ for all $n \in \mathbb{N}$. Let $\kappa_{1}(n)=2+\frac{\alpha_{n}}{\tau}$ and $\kappa_{2}(n)=2+\frac{\beta_{n}}{\tau}$. Then, by virtue of the resolvent identity and the nonexpansiveness of $J_{\alpha_{m}}^{A}$, one has for all $m \in \mathbb{N}$ that

$$
\begin{aligned}
\left\|J_{\alpha_{n}}^{A} x-J_{\alpha_{m}}^{A} x\right\| & =\left\|J_{\alpha_{m}}^{A}\left(\frac{\alpha_{m}}{\alpha_{n}} x+\left(1-\frac{\alpha_{m}}{\alpha_{n}}\right) J_{\alpha_{n}}^{A} x\right)-J_{\alpha_{m}}^{A} x\right\| \\
& \leq\left|1-\frac{\alpha_{m}}{\alpha_{n}}\right|\left\|J_{\alpha_{n}}^{A} x-x\right\| \leq\left(1+\frac{\alpha_{m}}{\alpha_{n}}\right)\left\|J_{\alpha_{n}}^{A} x-x\right\|,
\end{aligned}
$$

and thus

$$
\begin{aligned}
\left\|J_{\alpha_{m}}^{A} x-x\right\| & \leq\left\|J_{\alpha_{m}}^{A} x-J_{\alpha_{n}}^{A} x\right\|+\left\|J_{\alpha_{n}}^{A} x-x\right\| \\
& \leq\left(1+\frac{\alpha_{m}}{\alpha_{n}}+1\right)\left\|J_{\alpha_{n}}^{A} x-x\right\| \\
& \leq\left(2+\frac{\alpha_{m}}{\tau}\right)\left\|J_{\alpha_{n}}^{A} x-x\right\|=\kappa_{1}(m)\left\|J_{\alpha_{n}}^{A} x-x\right\|, \quad \forall n \geq m, \forall x \in \mathcal{H} .
\end{aligned}
$$


The same argument shows for all $m \in \mathbb{N}$ that

$$
\left\|J_{\beta_{m}}^{B} x-x\right\| \leq \kappa_{2}(m)\left\|J_{\beta_{n}}^{B} x-x\right\|, \quad \forall n \geq m, \forall x \in \mathcal{H}
$$

Therefore, condition (3.2) is true for $\left\{J_{\alpha_{n}}^{A}\right\}$ and $\left\{J_{\beta_{n}}^{B}\right\}$.

Putting $T_{n}=J_{\alpha_{n}}^{A}$ in Corollary 3.6 (resp. Corollary 3.7) and noting that $\{I\}$ and $\left\{J_{\alpha_{n}}^{A}\right\}$ verifies condition (3.2) due to $\liminf _{n \rightarrow \infty} \alpha_{n}>0$, we obtain the following two corollaries.

Corollary 4.6 Suppose that $A$ is a maximal monotone operator on $\mathcal{H}$ with $A^{-1} 0 \neq \varnothing$, and suppose that $\left\{a_{n}\right\},\left\{b_{n}\right\}$ and $\left\{c_{n}\right\}$ are sequences in $[0,1]$ with $a_{n}+b_{n}+c_{n}=1$ and $a_{n} \in(0,1)$ for all $n \in \mathbb{N}$. Let $\left\{\alpha_{n}\right\}$ be a sequence in $(0, \infty)$. For an arbitrary fixed $u \in \mathcal{H}$, choose an arbitrary $x_{1} \in \mathcal{H}$ and define

$$
x_{n+1}=a_{n} u+b_{n} x_{n}+c_{n} J_{\alpha_{n}}^{A} x_{n}, \quad n \in \mathbb{N} .
$$

Then the sequence $\left\{x_{n}\right\}$ converges strongly to $P_{A^{-1} 0} u$ provided the following conditions are satisfied:

$$
\lim _{n \rightarrow \infty} a_{n}=0, \quad \sum_{n=1}^{\infty} a_{n}=\infty, \quad \liminf _{n \rightarrow \infty} c_{n}>0, \quad \liminf _{n \rightarrow \infty} \alpha_{n}>0 .
$$

Corollary 4.7 [16] Suppose that $A$ is a maximal monotone operator on $\mathcal{H}$ with $A^{-1} 0 \neq \varnothing$, and suppose that $\left\{a_{n}\right\},\left\{b_{n}\right\}$ and $\left\{c_{n}\right\}$ are sequences in $[0,1]$ with $a_{n}+b_{n}+c_{n}=1$ and $a_{n} \in$ $(0,1)$ for all $n \in \mathbb{N}$. Let $\left\{\alpha_{n}\right\}$ be a sequence in $(0, \infty)$, and let $\left\{e_{n}\right\}$ be a bounded sequence in $\mathcal{H}$. For an arbitrary fixed $u \in \mathcal{H}$, choose an arbitrary $x_{1} \in \mathcal{H}$ and define

$$
x_{n+1}=a_{n} u+b_{n} x_{n}+c_{n} J_{\alpha_{n}}^{A} x_{n}+e_{n}, \quad n \in \mathbb{N} .
$$

Then the sequence $\left\{x_{n}\right\}$ converges strongly to $P_{A^{-1} 0} u$ provided the following conditions are satisfied:

$$
\begin{aligned}
& \text { (i) } \lim _{n \rightarrow \infty} a_{n}=0, \quad \sum_{n=1}^{\infty} a_{n}=\infty, \quad \liminf _{n \rightarrow \infty} c_{n}>0, \quad \liminf _{n \rightarrow \infty} \alpha_{n}>0 \text {; } \\
& \text { (ii) either } \lim _{n \rightarrow \infty} \frac{\left\|e_{n}\right\|}{a_{n}}=0 \text { or } \sum_{n=1}^{\infty}\left\|e_{n}\right\|<\infty .
\end{aligned}
$$

Let $f: \mathcal{H} \rightarrow(-\infty, \infty]$ be a proper lower semicontinuous convex function. The set of minimizers of $f$ is defined to be

$$
\underset{y \in \mathcal{H}}{\arg \min } f(y)=\{z \in \mathcal{H}: f(x) \leq f(y) \text { for all } y \in \mathcal{H}\},
$$

and the subdifferential of $f$ is defined as

$$
\partial f(x)=\{z \in \mathcal{H}:\langle y-x, z\rangle \leq f(y)-f(x), \forall y \in \mathcal{H}\}
$$


for all $x \in \mathcal{H}$. As shown in Rockafellar [23], $\partial f$ is a maximal monotone operator. Moreover, one has

$$
0 \in \partial f(z) \quad \Leftrightarrow \quad z \in \underset{y \in \mathcal{H}}{\arg \min } f(y)
$$

that is,

$$
(\partial f)^{-1} 0=\underset{y \in \mathcal{H}}{\arg \min } f(y) .
$$

Hence $\arg \min _{y \in \mathcal{H}} f(y)=F\left(J_{\alpha}^{\partial f}\right)$ for any $\alpha>0$, and then invoking Corollary 4.6, we obtain the following theorem.

Theorem 4.8 Let $f: \mathcal{H} \rightarrow(-\infty, \infty]$ be a proper lower semicontinuous convex function, and suppose that $\left\{a_{n}\right\},\left\{b_{n}\right\}$ and $\left\{c_{n}\right\}$ are sequences in $[0,1]$ with $a_{n}+b_{n}+c_{n}=1$ and $a_{n} \in$ $(0,1)$ for all $n \in \mathbb{N}$. Let $\left\{\alpha_{n}\right\}$ be a sequence in $(0, \infty)$, and put $\Omega=\arg \min _{y \in \mathcal{H}} f(y)$. For an arbitrary fixed $u \in \mathcal{H}$, choose an arbitrary $x_{1} \in \mathcal{H}$ and define

$$
x_{n+1}=a_{n} u+b_{n} x_{n}+c_{n} J_{\alpha_{n}}^{\partial f} x_{n} .
$$

Then the sequence $\left\{x_{n}\right\}$ converges strongly to $P_{\Omega} u$ provided the following conditions are satisfied:

$$
\begin{aligned}
& \text { (i) } \quad \lim _{n \rightarrow \infty} a_{n}=0, \quad \sum_{n=1}^{\infty} a_{n}=\infty \text {; } \\
& \text { (ii) } \quad \liminf _{n \rightarrow \infty} c_{n}>0, \quad \liminf _{n \rightarrow \infty} \alpha_{n}>0 \text {. }
\end{aligned}
$$

For any $n \in \mathbb{N}$, define $g_{n}: \mathcal{H} \rightarrow(-\infty, \infty]$ by

$$
g_{n}(z)=f(z)+\frac{1}{2 \alpha_{n}}\left\|z-x_{n}\right\|^{2}
$$

for all $z \in \mathcal{H}$. Then we have, for any $z \in \mathcal{H}$,

$$
\partial g_{n}(z)=\partial f(z)+\frac{1}{\alpha_{n}}\left(z-x_{n}\right),
$$

$c f$. [24]. Hence,

$$
\begin{aligned}
z \in\left(\partial g_{n}\right)^{-1} 0 & \Leftrightarrow \quad 0 \in \partial g_{n}(z) \\
& \Leftrightarrow \quad 0 \in \partial f(z)+\frac{1}{\alpha_{n}}\left(z-x_{n}\right) \\
& \Leftrightarrow \quad x_{n} \in z+\alpha_{n} \partial f(z)=\left(I+\alpha_{n} \partial f\right)(z) \\
& \Leftrightarrow \quad z=\left(I+\alpha_{n} \partial f\right)^{-1} x_{n}=J_{\alpha_{n}}^{\partial f} x_{n} .
\end{aligned}
$$


This means that $J_{\alpha_{n}}^{\partial f} x_{n}=\arg \min _{y \in \mathcal{H}} g_{n}(y)=\arg \min _{y \in \mathcal{H}}\left\{f(y)+\frac{1}{2 \alpha_{n}}\left\|y-x_{n}\right\|^{2}\right\}$, and thus the iterative scheme (23) is can be replaced with

$$
x_{n+1}=a_{n} u+b_{n} x_{n}+c_{n} \underset{y \in \mathcal{H}}{\arg \min }\left\{f(y)+\frac{1}{2 \alpha_{n}}\left\|y-x_{n}\right\|^{2}\right\} \text {. }
$$

Let $f: C \times C \rightarrow \mathbb{R}$. An equilibrium problem is the problem of finding $\hat{x} \in C$ such that

$$
f(\hat{x}, y) \geq 0, \quad \forall y \in C
$$

whose solution set is denoted by $\mathrm{EP}(f)$. For solving an equilibrium problem, we assume that the function $f$ satisfies the following conditions:

(A1) $f(x, x)=0, \forall x \in C$;

(A2) $f$ is monotone, that is, $f(x, y)+f(y, x) \leq 0, \forall x \in C$;

(A3) for all $x, y, z \in C$, $\lim \sup _{t \downarrow 0} f((1-t) x+t z, y) \leq f(x, y)$;

(A4) for all $x \in C, f(x, \cdot)$ is convex and lower semicontinuous.

The following lemma appears implicitly in Blum and Oetti [25] and is proved in detail by Aoyama et al. [26], while Lemma 4.10 is Lemma 2.12 of Combettes and Hirstoaga [27].

Lemma 4.9 [25,26] Let $f: C \times C \rightarrow \mathbb{R}$ be a function satisfying conditions (A1)-(A4), and let $r>0$ and $x \in \mathcal{H}$. Then there exists a unique $z \in C$ such that

$$
f(z, y)+\frac{1}{r}\langle y-z, z-x\rangle \geq 0, \quad \forall y \in C .
$$

Lemma 4.10 [27] Let $f: C \times C \rightarrow \mathbb{R}$ be a function satisfying conditions (A1)-(A4). For $r>0$, define $J_{r}^{f}: \mathcal{H} \rightarrow C$ by

$$
\int_{r}^{f} x=\left\{z \in C: f(z, y)+\frac{1}{r}\langle y-z, z-x\rangle \geq 0, \forall y \in C\right\}
$$

for all $x \in \mathcal{H}$. Then the following hold:

(a) $f_{r}^{f}$ is single-valued;

(b) $I_{r}^{f}$ is firmly nonexpansive;

(c) $F\left(f_{r}^{f}\right)=\mathrm{EP}(f)$;

(d) $\mathrm{EP}(f)$ is closed and convex.

We call $f_{r}^{f}$ the resolvent of $f$ for $r>0$. Using Lemmas 4.9 and 4.10, Takahashi et al. [15] established the lemma below.

Lemma 4.11 [15] Let $f: C \times C \rightarrow \mathbb{R}$ be a function satisfying conditions (A1)-(A4) and define a set-valued mapping of $\mathcal{H}$ into itself by

$$
G_{f}(x)= \begin{cases}\{z \in \mathcal{H}: f(x, y) \geq\langle y-x, z\rangle, \forall y \in C\}, & \forall x \in C, \\ \varnothing, & \forall x \notin C .\end{cases}
$$

Then the following hold:

(a) $G_{f}$ is a maximal monotone operator with $\mathcal{D}\left(G_{f}\right) \subseteq C$; 
(b) $\mathrm{EP}(f)=G_{f}^{-1} 0$;

(c) $J_{r}^{G_{f}} x=J_{r}^{f} x$ for all $x \in \mathcal{H}$.

Theorem 4.12 Let $C$ be a nonempty closed convex subset of $\mathcal{H}$ and let $f_{i}: C \times C \rightarrow \mathbb{R}$, $i=1,2$, be functions satisfying conditions (A1)-(A4) with $\operatorname{EP}\left(f_{1}\right) \cap \operatorname{EP}\left(f_{2}\right) \neq \varnothing$. Suppose that $\left\{a_{n}\right\},\left\{b_{n}\right\},\left\{c_{n}\right\}$ and $\left\{d_{n}\right\}$ are sequences in $[0,1]$ with $a_{n}+b_{n}+c_{n}+d_{n}=1$ and $a_{n} \in(0,1)$ for all $n \in \mathbb{N}$. Let $\left\{\alpha_{n}\right\}$ and $\left\{\beta_{n}\right\}$ be sequences in $(0, \infty)$, and let $\left\{e_{n}\right\}$ be a bounded sequence in $\mathcal{H}$. For an arbitrary fixed $u \in \mathcal{H}$, choose an arbitrary $x_{1} \in \mathcal{H}$ and define

$$
x_{n+1}=a_{n} u+b_{n} J_{\beta_{n}}^{f_{2}} x_{n}+c_{n} J_{\alpha_{n}}^{f_{1}} x_{n}+d_{n} e_{n}, \quad n \in \mathbb{N} .
$$

Then the sequence $\left\{x_{n}\right\}$ converges strongly to $P_{\mathrm{EP}\left(f_{1}\right) \cap \mathrm{EP}\left(f_{2}\right)}$ u provided the following conditions are satisfied:

(i) $\quad \lim _{n \rightarrow \infty} a_{n}=\lim _{n \rightarrow \infty} \frac{d_{n}}{a_{n}}=0, \quad \sum_{n=1}^{\infty} a_{n}=\infty, \quad \sum_{n=1}^{\infty} d_{n}<\infty$;

(ii) $\quad \liminf _{n \rightarrow \infty} b_{n}>0, \quad \liminf _{n \rightarrow \infty} c_{n}>0$;

(iii) $\quad \liminf _{n \rightarrow \infty} \alpha_{n}>0, \quad \liminf _{n \rightarrow \infty} \beta_{n}>0$.

Proof The set-valued mappings $G_{f_{i}}$ associated with $f_{i}, i=1,2$, defined in Lemma 4.11 are maximal monotone operators with $\mathcal{D}\left(G_{f_{i}}\right) \subseteq C$, and it follows from Lemmas 4.10 and 4.11 that $J_{r}^{G_{f_{i}}}=J_{r}^{f_{i}}$ and $F\left(J_{r}^{G_{f_{i}}}\right)=F\left(f_{r}^{f_{i}}\right)=\operatorname{EP}\left(f_{i}\right)=G_{f_{i}}^{-1} 0$ for any $r>0$. Putting $B=G_{f_{2}}$ and $A=G_{f_{1}}$ in Theorem 4.5, the desired conclusion follows.

Here, it is worth mentioning, just as the SFP, that the unique minimum norm solution can be obtained through our algorithm for each of the minimization problem and the equilibrium problem by taking $u=0$ in Theorems 4.8 and 4.12 .

For a nonempty closed convex subset $C$ of $\mathcal{H}$, its indicator function $\iota_{C}$ defined by

$$
\iota_{C}(x)= \begin{cases}0, & x \in C ; \\ \infty, & x \notin C,\end{cases}
$$

is a proper lower semicontinuous convex function and its subdifferential $\partial \iota_{C}$ defined by

$$
\partial \iota_{C}(x)=\left\{z \in \mathcal{H}:\langle y-x, z\rangle \leq \iota_{C}(y)-\iota_{C}(x), \forall y \in \mathcal{H}\right\}
$$

is a maximal monotone operator, $c f$. Rockafellar [23]. As shown in Lin and Takahashi [28], the resolvent $J_{r}^{\partial \iota_{C}}$ of $\partial \iota_{C}$ for $r>0$ is the same as the metric projection $P_{C}$, and $\left(\partial \iota_{C}\right)^{-1} 0=C$.

Theorem 4.13 Let $C_{i}, i=1,2$, be two nonempty closed convex subsets of $\mathcal{H}$ with $C_{1} \cap$ $C_{2} \neq \varnothing$. Suppose that $\left\{a_{n}\right\},\left\{b_{n}\right\},\left\{c_{n}\right\}$ and $\left\{d_{n}\right\}$ are sequences in $[0,1]$ with $a_{n}+b_{n}+c_{n}+d_{n}=1$ and $a_{n} \in(0,1)$ for all $n \in \mathbb{N}$. Let $\left\{e_{n}\right\}$ be a bounded sequence in $\mathcal{H}$. For an arbitrary fixed $u \in \mathcal{H}$, choose an arbitrary $x_{1} \in \mathcal{H}$ and define

$$
x_{n+1}=a_{n} u+b_{n} P_{C_{2}} x_{n}+c_{n} P_{C_{1}} x_{n}+d_{n} e_{n}, \quad n \in \mathbb{N} .
$$


Then the sequence $\left\{x_{n}\right\}$ converges strongly to $P_{C_{1} \cap C_{2}} u$ provided the following conditions are satisfied:

(i) $\quad \lim _{n \rightarrow \infty} a_{n}=\lim _{n \rightarrow \infty} \frac{d_{n}}{a_{n}}=0, \quad \sum_{n=1}^{\infty} a_{n}=\infty, \quad \sum_{n=1}^{\infty} d_{n}<\infty$;

(ii) $\quad \liminf _{n \rightarrow \infty} b_{n}>0, \quad \liminf _{n \rightarrow \infty} c_{n}>0$.

Proof Putting $A=\partial \iota_{C_{1}}$ and $B=\partial \iota_{C_{2}}$ in Theorem 4.5, the desired conclusion follows.

\section{Competing interests}

The authors declare that they have no competing interests.

\section{Authors' contributions}

Both authors contributed equally to this work. Both authors read and approved the final manuscript.

\section{Author details}

'Department of Accounting Information, Southern Taiwan University of Science and Technology, 1 Nantai St., Yongkang Dist., Tainan, 71005, Taiwan. ${ }^{2}$ Department of Industrial Management, National Pingtung University of Science and Technology, 1 Shuefu Rd., Neipu, Pingtung, 91201, Taiwan.

\section{Acknowledgements}

The work was supported by the National Science Council of Taiwan with contract No. NSC101-2221-E-020-031.

Received: 18 April 2013 Accepted: 5 July 2013 Published: 19 July 2013

\section{References}

1. Censor, Y, Elfving, T: A multiprojection algorithm using Bregman projection in a product space. Numer. Algorithms 8 , 221-239 (1994)

2. Xu, HK: Iterative methods for the split feasibility problem in infinite-dimensional Hilbert spaces. Inverse Probl. 26, 105018 (2010). doi:10.1088/0266-5611/26/10/105018

3. Byrne, C: Iterative oblique projection onto convex subsets and the split feasibility problem. Inverse Probl. 18, 441-453 (2002)

4. Byrne, $\mathrm{C}:$ A unified treatment of some iterative algorithms in signal processing and image reconstruction. Inverse Probl. 20, 103-120 (2004)

5. Yao, Y, Jigang, W, Liou, YC: Regularized methods for the split feasibility problem. Abstr. Appl. Anal. (2012). doi:10.1155/2012/140679

6. Yao, Y, Liou, YC, Shahzad, N: A strongly convergent method for the split feasibility problem. Abstr. Appl. Anal. (2012). doi:10.1155/2012/125046

7. Martinet, B: Régularisation d'inéquations variationnelles par approximations successives. Rev. Fr. Inform. Rech. Oper. 4, 154-158 (1970)

8. Rockafellar, RT: Monotone operators and the proximal point algorithm. SIAM J. Control Optim. 14, $877-898$ (1976)

9. Güler, O: On the convergence of the proximal point algorithm for convex minimization. SIAM J. Control Optim. 29, 403-419 (1991)

10. Boikanyo, OA, Moroşanu, G: Inexact Halpern-type proximal point algorithm. J. Glob. Optim. 51, $11-26$ (2011)

11. Boikanyo, OA, Moroşanu, G: Four parameter proximal point algorithms. Nonlinear Anal. 74, 544-555 (2011)

12. Boikanyo, OA, Moroşanu, G: A proximal point algorithm converging strongly for general errors. Optim. Lett. 4, 635-641 (2010). doi:10.1007/s11590-010-0176-z

13. Kamimura, S, Takahashi, W: Approximating solutions of maximal monotone operators in Hilbert spaces. J. Approx. Theory 106, 226-240 (2000)

14. Solodov, MV, Svaiter, BF: Forcing strong convergence of proximal point iterations in a Hilbert space. Math. Program. 87, 189-202 (2000)

15. Takahashi, S, Takahashi, W, Toyoda, M: Strong convergence theorems for maximal monotone operators with nonlinear mappings in Hilbert spaces. J. Optim. Theory Appl. 147, 27-41 (2010)

16. Wang, F, Cui, H: On the contraction-proximal point algorithms with multi-parameters. J. Glob. Optim. 54, 485-491 (2012) doi:10.1007/s10898-011-9772-4

17. Xu, HK: A regularization method for the proximal point algorithm. J. Glob. Optim. 36, 115-125 (2006) doi:10.1007/s10898-006-9002-7

18. Xu, HK: Iterative algorithms for nonlinear operators. J. Lond. Math. Soc. 66(2), 240-256 (2002)

19. Yao, Y, Noor, MA: On convergence criteria of generalized proximal point algorithm. J. Comput. Appl. Math. 217, 46-55 (2008)

20. Marino, G, Xu, HK: Convergence of generalized proximal point algorithm. Commun. Pure Appl. Anal. 3, 791-808 (2004)

21. Goebel, K, Kirk, WA: Topics in Metric Fixed Point Theory. Cambridge University Press, Cambridge (1990)

22. Maingé, PE: Strong convergence of projected subgradient methods for nonsmooth and nonstrictly convex minimization. Set-Valued Anal. 16, 899-912 (2008). doi:10.1007/s11228-008-0102-z 
23. Rockafellar, RT: On the maximal monotonicity of subdifferential mappings. Pac. J. Math. 33, 209-216 (1970)

24. Takahashi, W: Introduction to Nonlinear and Convex Analysis. Yokohama Publishers, Yokohama (2009)

25. Blum, E, Oettli, W: From optimization and variational inequalities to equilibrium problems. Math. Stud. 63, 123-145 (1994)

26. Aoyama, K, Kimura, T, Takahashi, W: Maximal monotone operators and maximal monotone functions for equilibrium problems. J. Convex Anal. 15, 395-409 (2008)

27. Combettes, PL, Hirstoaga, SA: Equilibrium programming in Hilbert spaces. J. Nonlinear Convex Anal. 6, 117-136 (2005)

28. Lin, LJ, Takahashi, W: A general iterative method for hierarchical variational inequality problems in Hilbert spaces and applications. Positivity 16, 429-453 (2012)

doi:10.1186/1687-1812-2013-190

Cite this article as: Huang and Hong: Approximating common fixed points of averaged self-mappings with applications to the split feasibility problem and maximal monotone operators in Hilbert spaces. Fixed Point Theory and Applications 2013 2013:190

\section{Submit your manuscript to a SpringerOpen ${ }^{\circ}$ journal and benefit from:}

- Convenient online submission

- Rigorous peer review

- Immediate publication on acceptance

- Open access: articles freely available online

- High visibility within the field

- Retaining the copyright to your article 